\title{
Efeito da Ordem de Adição de Componentes nas Propriedades de Misturas NR/BR
}

\author{
Daniele F. Castro, Regina C. R. Nunes, Leila L. Y. Visconte \\ Instituto de Macromoléculas, UFRJ
}

\author{
João C. M. Suarez \\ Departamento de Engenharia Mecânica e de Materiais, Instituto Militar de Engenharia, RJ
}

\begin{abstract}
Resumo: Misturas de elastômeros vêm sendo largamente utilizadas na indústria de borracha com a finalidade de se conseguir propriedades específicas, sem a necessidade de sintetizar novos polímeros. O polibutadieno puro (BR) não apresenta boas características de processamento e de resistência à tração e ao rasgamento sendo, por essa razão, normalmente utilizado em misturas com outros elastômeros, principalmente NR e SBR. Tais misturas são amplamente utilizadas na indústria de pneus. Neste trabalho o polibutadieno (BR) foi misturado à borracha natural (NR) na proporção de 1:1 em peso. As composições foram preparadas em misturador de cilindros, empregando-se quatro maneiras distintas de incorporação dos aditivos. Após a vulcanização, foram estudadas as propriedades mecânicas (resistência à tração, ao rasgamento e dureza) e a morfologia das composições. Os resultados mostram que, apesar de apresentarem a mesma formulação, as propriedades das misturas são bastante influenciadas pelo modo de preparo.
\end{abstract}

Palavras-chave: Mistura de elastômeros, propriedades mecânicas, análise morfológica.

\section{Properties of NR/BR Mixtures and their Dependence on the Preparation Mode}

Abstract: Elastomer Blends have been widely used in the rubber industry with the purpose of reaching specific properties without the need of synthesizing new polymers. Polybutadiene rubber (BR) presents poor stress and tear strengths, which is the main reason why BR is usually blended with NR and SBR. These blends are extensively used in the manufacture of tyres. In this work natural rubber (NR) was mixed to polybutadiene rubber (BR) in a 50:50 w/w ratio. The compositions were prepared in a two-roll mill employing four different modes of introducing the additives. After vulcanization, the mixtures were studied with evaluation of their mechanical (stress and tear strengths and hardness) properties and morphology. The results show that the properties varied even using the same NR/BR formulations due to the different preparation techniques.

Keywords: Elastomer blends, mechanical properties, morphology.

\section{Introdução}

O uso de apenas um elastômero muitas vezes não é suficiente para se obter determinadas propriedades exigidas na aplicação de artefatos de borracha; torna-se então, necessária a mistura de dois ou mais elastômeros. Deste modo, produtos na indústria de borracha são total ou parcialmente baseados em misturas de elastômeros ${ }^{[1-3]}$.

Misturas de elastômeros são feitas principalmente por três razões: melhorar as propriedades dos elastômeros originais, melhorar o processamento e diminuir o custo ${ }^{[2-4]}$. Essas misturas possibilitam que se consiga um balanço de propriedades onde as vantagens de um elastômero compensem as desvantagens do outro e vice-versa ${ }^{[5,6]} \mathrm{e}$, desse modo, ampliam a faixa de propriedades atendidas sem que novos polímeros tenham que ser sintetizados, e sim sejam empregados elastômeros já conhecidos e bem caracterizados ${ }^{[7]}$. Misturas poliméricas são normalmente sistemas de duas fases e a distribuição dos aditivos por essas fases não é necessariamente uniforme, já que os ingredientes adicionados às misturas têm diferentes solubilidades em cada um dos elastômeros, e estarão em maior concentração na fase com a qual apresentem maior afinidade, o que acabará afetando a velocidade e o grau de vulcanização das composições. Mesmo com uma distribuição uniforme dos ingredientes, os elastômeros da mistura dificilmente vulcanizam a razões idênticas, já que suas reatividades frente à reação de vulcanização com enxofre são afetadas pela estrutura química, peso molecular e conformação do elastômero base ${ }^{[3,8]}$.

Nos últimos anos, tem havido um interesse crescente por misturas de borrachas para uso em uma grande variedade de produtos comerciais ${ }^{[7]}$. Um setor da indústria no qual elas vêm sendo largamente empregadas é na produção de pneus, onde misturas, principalmente, de NR/BR e NR/SBR são bastante utilizadas ${ }^{[1,2,7,9]}$. 
Neste trabalho, foram feitas misturas de borracha natural (NR), que tem uma excelente resistência à tração e boa resistência ao rasgamento, pois pode cristalizar por estiramento ${ }^{[4,6,10]}$ e de polibutadieno (BR), que apesar de apresentar ótima resistência à abrasão, quando utilizado puro, geralmente apresenta características pobres de processabilidade e rasgamento sendo, no entanto, muito utilizado em misturas, porque contribui em várias propriedades em artigos vulcanizados como: melhoria na resistência à abrasão, à fadiga e ao envelhecimento ${ }^{[10,11]}$. As misturas foram feitas na proporção de 1:1 em peso, utilizandose quatro maneiras diferentes de incorporação das borrachas e dos aditivos e o efeito do modo de preparo sobre as propriedades mecânicas e a morfologia foram investigados.

\section{Experimental}

A borracha natural (NR) e o polibutadieno (BR) foram adicionados na proporção de 1:1 em peso. A seguinte formulação foi utilizada na preparação das misturas (em phr): NR (elastômero) (50); BR (elastômero) (50); óxido de zinco (ativador) (3,0); ácido esteárico (ativador) (2,5); aminox (antioxidante), produto da reação, a baixa temperatura, entre difenilamina e acetona $(2,0)$; PVI (inibidor), N-ciclohexiltioftalimida $(0,3)$; enxofre (agente de vulcanização) $(2,5)$; TBBS (acelerador), t-butil-2-benzotiazol sulfenamida $(0,6)^{[12]}$. As misturas foram preparadas em misturador de cilindros Berstorff, a $50{ }^{\circ} \mathrm{C}$, empregando quatro modos diferentes para a incorporação dos aditivos:

- M1: os aditivos, com exceção do acelerador, foram misturados inicialmente na borracha natural e, após a homogeneização, o polibutadieno foi adicionado. Por último, à mistura homogênea, acrescentou-se o acelerador;

- M2: os aditivos, com exceção do acelerador, foram misturados inicialmente ao polubutadieno e, após a homogeneização a borracha natural foi adicionada. Por último, à mistura homogênea, acrescentou-se o acelerador;

- M3: as borrachas foram previamente misturadas, antes da adição dos aditivos; e

- M4: a cada uma das borrachas foi adicionada metade da quantidade de cada um dos aditivos, com exceção do acelerador. As composições das duas borrachas foram então misturadas e, após a homogeneização, o acelerador foi adicionado.

Em seguida foi feita a determinação dos parâmetros reométricos, utilizando reômetro de disco oscilatório, da marca Tecnología Industrial, modelo TI-100, operando com arco de $3^{\circ}$ e uma temperatura de $160{ }^{\circ} \mathrm{C}$. Os ensaios de resistência à tração (ASTM D 412) e resistência ao rasgamento (ASTM D 624) foram realizados em dinamômetro Instron, modelo 1101, a temperatura ambiente e com velocidade de deformação de $500 \mathrm{~mm} / \mathrm{min}$. O ensaio de dureza (ASTM D 2240) foi feito em Durômetro Shore Tipo A-2. Os corpos de prova, das diferentes composições, específicos para tais ensaios, foram cortados de placas vulcanizadas a partir dos valores de $\mathrm{t}_{90}$ (tempo de vulcanização a $90 \%$ de cura).
A morfologia das misturas foi examinada por microscopia eletrônica de varredura (SEM), utilizando-se um microscópio Jeol, modelo JSM 5800LV. A análise microscópica foi realizada pela observação direta da topografia de superfícies de fratura, resultantes da quebra mecânica de amostras obtidas por meio de cada um dos modos de mistura, após sua imersão em nitrogênio líquido. Antes do exame microscópico, as superfícies de fratura foram recobertas com uma fina camada de ouro, em câmara à vácuo.

\section{Resultados e Discussão}

A Tabela 1 apresenta os parâmetros reométricos para as borrachas puras e para as quatro misturas. Em relação ao comportamento das borrachas puras, a Tabela 1 confirma a maior reatividade da NR para a formação de ligações cruzadas do que o BR, o que faz com que a NR apresente menores valores de $t_{90}$ e $t_{s 2}$, e maior índice de velocidade de cura (CRI). Comparando-se as composições com misturas de borrachas, percebe-se que o modo de preparo tem influência sobre o tempo ótimo de cura, com as composições M2 e M3 apresentando valores bem próximos ao da NR, embora para todas elas o valor de $t_{90}$ seja intermediário entre os valores encontrados para as borrachas puras.

Como a NR vulcaniza muito mais rápido que o $\mathrm{BR}$, o que pode ser visto também na Figura 1, observa-se que quanto

Tabela 1. Propriedades reométricas das misturas NR/BR.

\begin{tabular}{clcccr}
\hline Material & $\begin{array}{c}\mathbf{t}_{\mathbf{9 0}} \\
(\mathbf{m i n})\end{array}$ & $\begin{array}{c}\text { ML } \\
(\mathbf{l b . i n})\end{array}$ & $\begin{array}{c}\text { MH } \\
(\mathbf{l b . i n})\end{array}$ & $\begin{array}{c}\mathbf{t}_{\mathbf{s} 2} \\
(\mathbf{m i n})\end{array}$ & $\mathbf{C R I}$ \\
\hline NR & 10,59 & 8,25 & 49,95 & 5,94 & 20,61 \\
BR & 35,5 & 12,7 & 49,35 & 13,5 & 4,50 \\
M1 & 14,59 & 6,6 & 52,9 & 6,81 & 12,85 \\
M2 & 11,29 & 7,7 & 54,6 & 6,66 & 21,59 \\
M3 & 11 & 8,0 & 54,9 & 6,04 & 20,20 \\
M4 & 13,41 & 7,55 & 46 & 7,11 & 15,87 \\
\hline
\end{tabular}

$\mathrm{t}_{90}$ : tempo ótimo de cura; $\mathrm{M}_{\mathrm{L}}$ : torque mínimo; $\mathrm{M}_{\mathrm{H}}$ : torque máximo; $\mathrm{t}_{\mathrm{s} 2}:$ tempo de pré-vulcanização; e CRI: índice de cura.

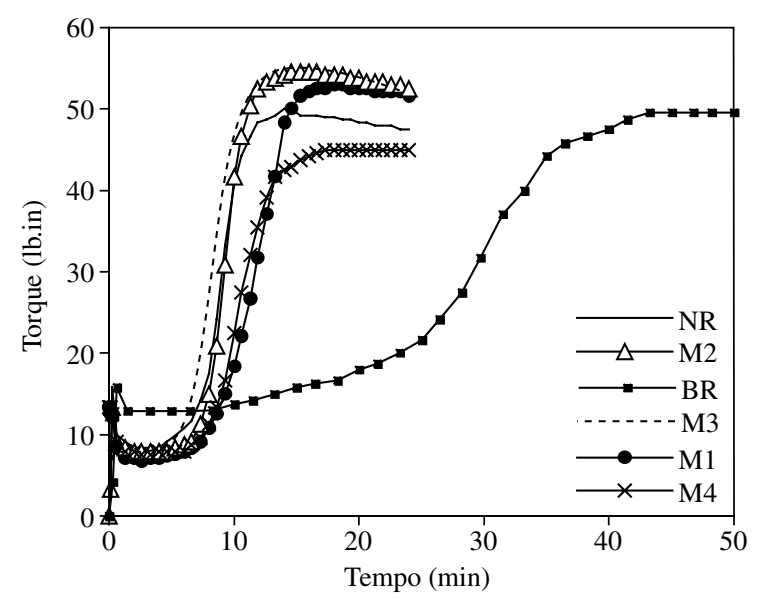

Figura 1. Curvas reométricas das misturas NR/BR. 
mais alto o valor de $t_{90}$ encontrado para as misturas, maior será a tendência da NR começar a sofrer degradação termooxidativa por causa do aquecimento prolongado. No entanto, quanto menores forem esses períodos de tempo, menor será o grau de vulcanização da fase BR. Esses dois fatores combinados permitem prever que as misturas não terão bom desempenho mecânico.

A degradação termo-oxidativa da NR ocorre normalmente pela cisão ou despolimerização do elastômero, tornando-o gradativamente mais macio e pegajoso e diminuindo assim o seu torque máximo, o qual está relacionado com o número de ligações cruzadas. Em relação ao torque máximo, as borrachas puras apresentam valores próximos e as misturas, com exceção da composição M4, valores que, além de não variarem muito entre si, são superiores aos das borrachas puras. A Figura 1 permite perceber ainda que as misturas M1, M2 e M3, assim como a borracha natural pura, apresentam diminuição do torque máximo após atingirem um valor máximo, o que indica que estas composições estão passando por um processo de degradação da NR. É possível que os produtos da cisão auxiliem a vulcanização do BR. Já a mistura M4 não apresenta redução do torque máximo, o que sugere que, ou esteja havendo degradação da NR segundo o mecanismo de oxidação das ligações carbono-carbono, no qual os radicais livres gerados poderiam atacar as cadeias de polímero levando a um aumento do número de ligações cruzadas, e tornando o material duro e quebradiço; ou, uma outra possibilidade seria a ocorrência de uma ação protetora do BR que dificultaria a degradação da NR, ou ainda os dois casos.

A semelhança entre os parâmetros reométricos para M2 e M3 sugere que ocorre uma migração de aditivos de NR para $\mathrm{BR}$, indicativo de uma maior afinidade dos aditivos com o polibutadieno.

As Figuras 2 e 3 apresentam os resultados de propriedades mecânicas para as duas borrachas puras e para as quatro misturas.

Os resultados de resistência à tração apresentados na Figura 2a, confirmam a superioridade da NR em relação ao BR. Já as misturas, apresentam valores intermediários aos das borrachas puras, e são dependentes do modo de preparo, sendo maior para M2 e menor para M1. Na mistura M2 a vulcanização da fase BR é estimulada, uma vez que todos os aditivos são incorporados nesta fase obtendo-se, dessa forma, uma relativa proteção à fase NR. Como o $t_{90}$ dessa mistura é próximo ao da NR, não ocorre uma acentuada degradação desta, o que resulta em valor de resistência à tração superior aos das demais misturas. Ao contrário, na mistura M1, a presença dos vulcanizantes na fase NR provoca cura excessiva desta fase podendo provocar um alto grau de degradação da mesma, além de prejudicar a vulcanização do BR, resultando no baixo valor desta propriedade.

Quanto à resistência ao rasgamento, a Figura $2 \mathrm{~b}$ mostra que o modo de preparo não tem a mesma influência observada para a resistência à tração. M2 continua apresentando o melhor resultado; no entanto M3, que apresentava boa re- sistência à tração, tem o pior valor de rasgamento, embora a variação entre os resultados seja pequena.

A Figura 2a mostra ainda que o alongamento na ruptura da borracha natural é muito superior ao do polibutadieno, confirmando sua maior flexibilidade. Já as misturas apresentam, conforme esperado, valores intermediários aos das borrachas puras, sendo o melhor resultado apresentado pela M2, o que pode ser resultado do menor valor de $\mathrm{t}_{90}$ usado na vulcanização desta mistura, contribuindo para uma menor degradação da fase NR.

Pode-se observar na Figura 3 que, conforme o esperado, os valores de dureza das borrachas puras e das misturas são muito próximos e que o modo de preparação das misturas não tem influência significativa sobre esta propriedade.

Como pôde ser observado pelas propriedades dos elastômeros puros, a NR é muito superior ao BR. A mistura dos

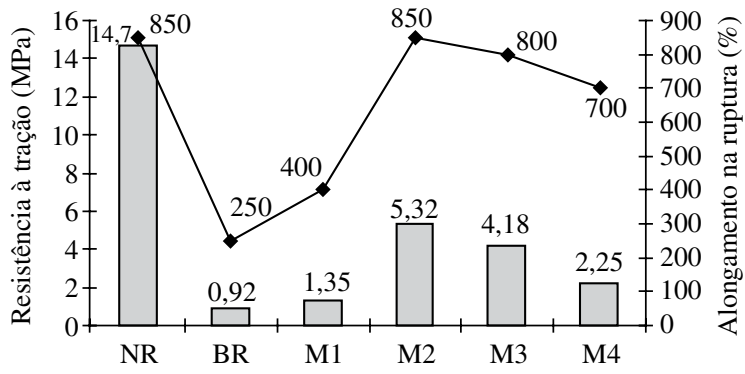

(a) Resistência à tração e alongamento na ruptura

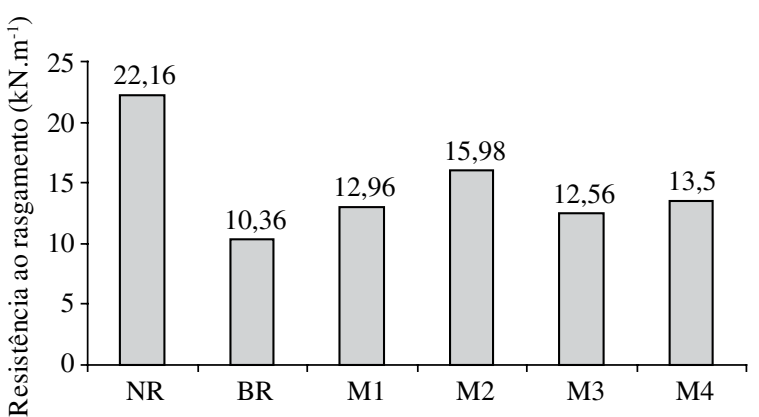

(b) Resistência ao rasgamento

Figura 2. Propriedades Mecânicas das misturas NR/BR

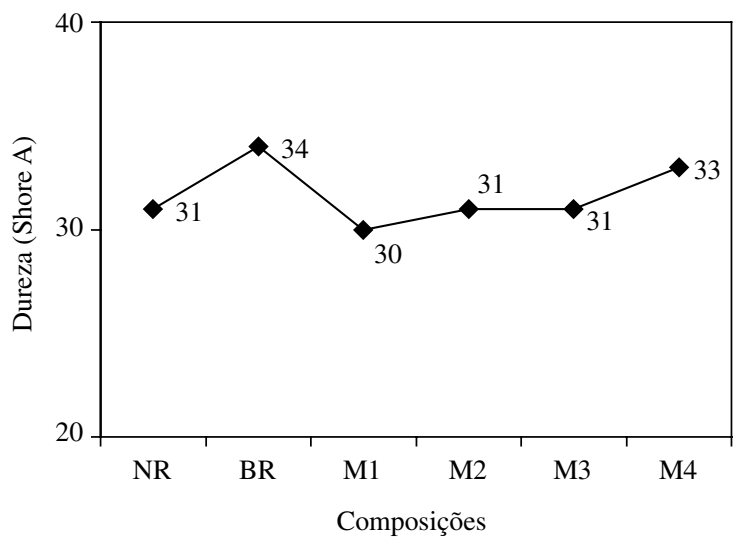

Figura 3. Dureza das misturas NR/BR. 
dois, apesar de apresentar resultados bastante inferiores aos da NR, foi vantajosa pois, em todos os quatro casos, houve uma melhora na processabilidade que no caso do BR puro é muito ruim e por essa razão, raramente é usado nesta forma. O menor preço da NR, em comparação ao do BR, também é um fator positivo na realização das misturas, pois é possível melhorar as propriedades das composições do BR, ao mesmo tempo em que se reduz o custo das mesmas.

Os aspectos microscópicos das superfícies criofraturadas das amostras M1 a M4, observadas com pequeno aumento, estão apresentados na Figura 4. Verifica-se a existência de distintos elementos topográficos, indicando que o comportamento na fratura da mistura NR/BR 50/50 pode ser afetado por variações nas condições de processamento. Microfratografias das amostras M1 a M4, com maiores aumentos, são apresentadas na Figura 5.

$\mathrm{Na}$ amostra M1 podem ser observados elementos característicos de um mecanismo misto de fratura, com regiões planas associadas a locais com deformação plástica localizada e áreas de rasgamento (Figura 4a). Em maiores aumentos verifica-se que a região plana apresenta a existência de uma grande quantidade de trincas que se desenvolvem paralelamente (Fi-

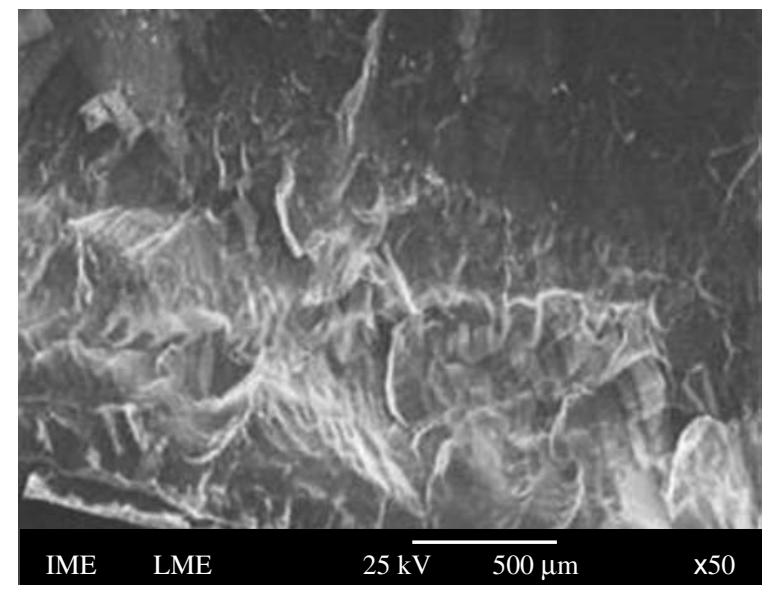

(a)

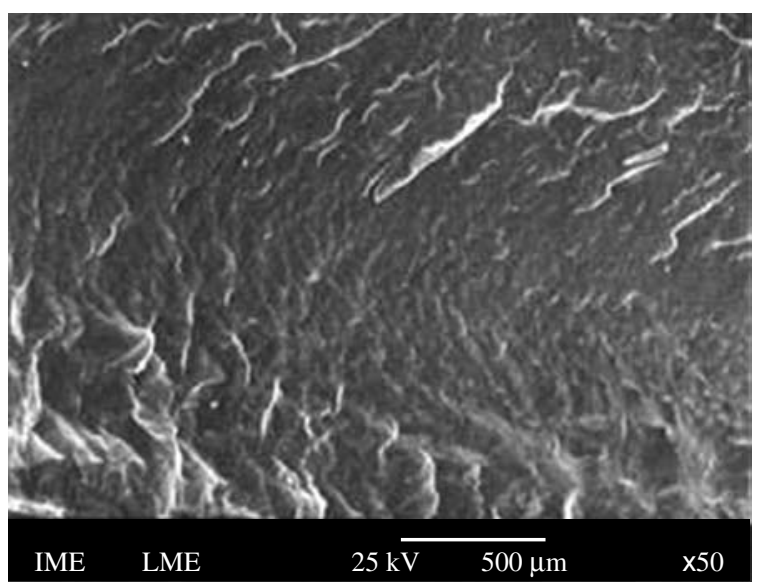

(c) gura 5a), caracterizando uma menor plasticidade do material.

As amostras M2 e M3 mostram aspectos microscópicos bastante semelhantes (Figuras $4 b$ e $4 c$ ), com superfícies de fratura que apresentam pequena rugosidade superficial e estrias, caracterizando uma fratura mais dúctil. A amostra M2 apresenta, em relação à amostra $\mathrm{M} 3$, uma maior quantidade de estrias (Figuras 5b e 5c) mostrando a sua maior capacidade de deformação plástica.

A amostra M4 apresenta uma superfície de fratura parcialmente dúctil, mostrando regiões de cisalhamento na parte próxima à superfície lateral e uma região rugosa grosseira na parte central (Figura 4d). Em maiores aumentos verifica-se que a região central apresenta facetas (Figura 5d), indicando a ocorrência de um processo descontínuo de fratura.

As observações obtidas por microscopia estão de acordo com os resultados dos ensaios mecânicos.

\section{Conclusões}

O modo de preparo das misturas tem uma grande influência sobre seu comportamento, tornando possível conseguir adequar determinadas propriedades, sem alterar a formulação.

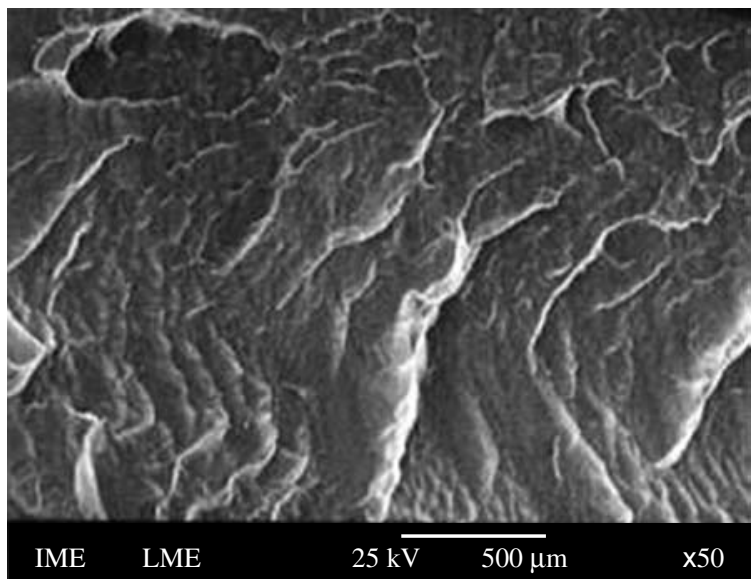

(b)

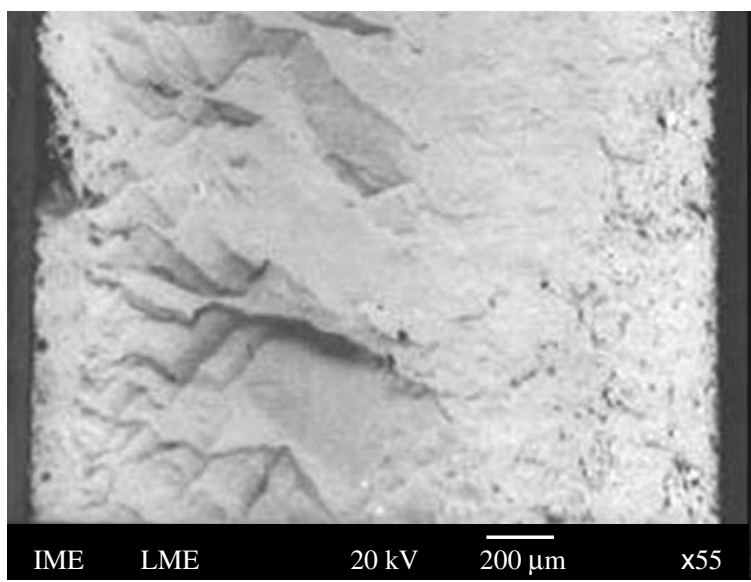

(d)

Figura 4. Fotomicrografias das misturas NR/BR, 25 kV, 50x: a) M1; b) M2; c) M3 e 20 kV, 55x; e d) M4. 


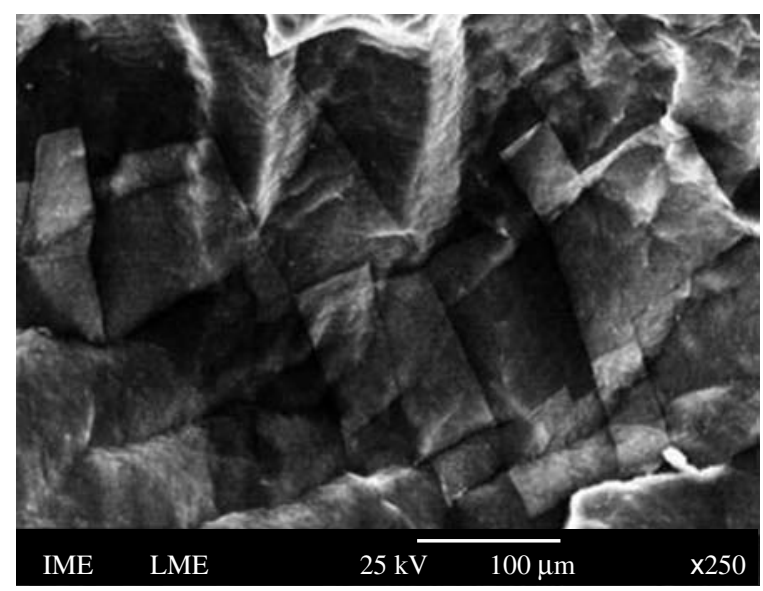

(a)

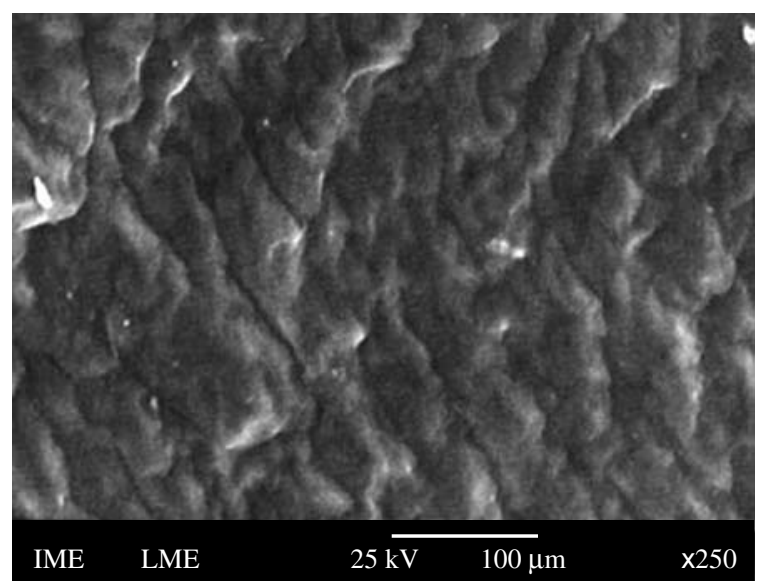

(c)

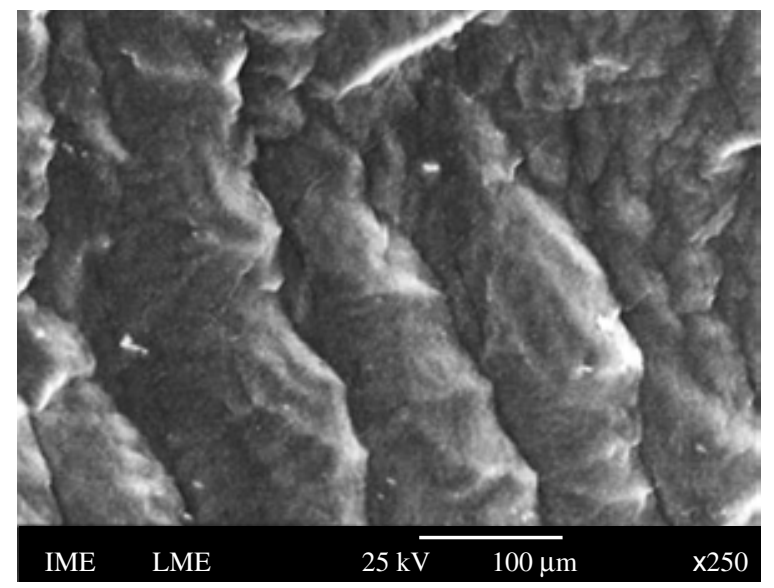

(b)

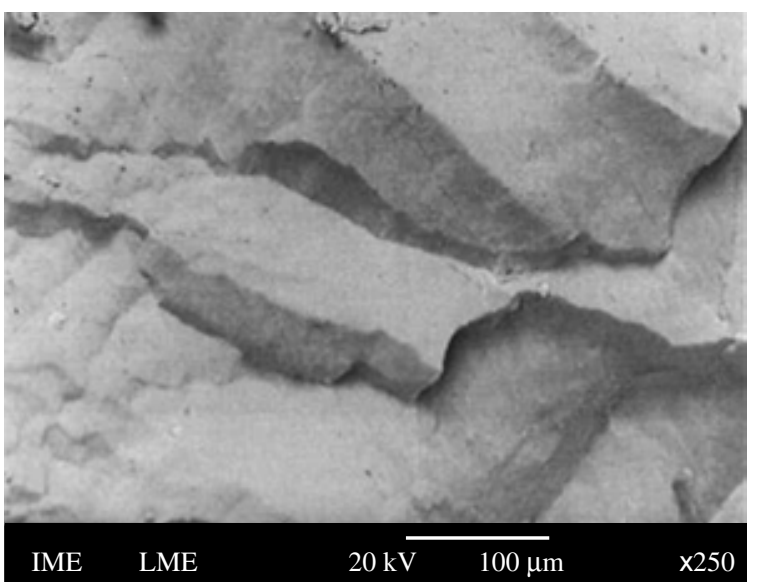

(d)

Figura 5. Fotomicrografias das misturas NR/BR, 25 kV, 250x: a) M1; b) M2; c) M3 e 20 kV, 250x; e d) M4.

No caso das misturas NR/BR, os melhores resultados foram obtidos quando se favorece a vulcanização do polibutadieno, preservando-se a NR da cura excessiva e da sua posterior degradação.

O comportamento na fratura da mistura NR/BR 50/50 é influenciado pelas suas condições de processamento, que provavelmente afetam a estrutura molecular do material. Diferentes morfologias da mistura podem estar associadas com diferentes histórias de processamento.

\section{Agradecimentos}

Os autores agradecem à Petroflex Indústria e Comércio S.A. pela doação do polibutadieno, à Teadit pela doação da Borracha Natural e ao IME pela realização das microscopias.

\section{Referências Bibliográficas}

1. Joseph, R.; George, K.E.; Francis, D.J. \& Thomas, K.T. - Int. J. Polym Mater, 12, p.53 (1987).

2. Joseph, R.; George, K.E. \& Francis, D.J - Int. J. Polym. Mater, 12, p.111 (1988).

3. Huson, M.G.; McGill, W.J. \& Swart, P.J. - Journal of Polymer Science Polymer Letters Edition, 22, p.143 (1984).
4. Corish, P.J. - "Elastomer Blends", in: Science and Technology of Rubber, J.E. Mark, B. Herman e F. R. Eirich (ed), Academic Press, Inc., San Diego, Califórrnia (1994).

5. Ultracki, L.A. - "Polymer Alloys and Blends: Thermodynamic and Rheology", Hanser Publishes, New York (1990).

6. Costa, V.G. \& Nunes, R.C. - Eur. Polym. J., 30, p.1025 (1994).

7. Klei, B. \& Koenig, J.L. - Rubber Chem. Technol, 70, p.231 (1997).

8. Chough, S. \& Chang, D. - Journal of Applied Polymer Science, 61, p.449 (1996).

9. Hurston, D.J. \& Song, M. - Journal of Applied Polymer Science, 76, p.1791 (2000).

10. Thorn, A.D. \& Robinson, R.A. - "Compound Design" in: Rubber Products Manufacturing Technology, cap.1, Bhowmich A.N.K, Hall M.M. \& Bernarey H.A. (ed), Marcel Dekker Inc., New York (1994).

11. Leutewiler, C.L. - Borracha Atual, 34 (2001).

12. Wampler, W.A., Gerspacher, M., Yang H.H. \& O'Farrell, C. P. - paper $n^{\circ} 52$ presented to Rubber Division, American Chemical Society, Pittsburgh, Pennsylvania (1994).

Enviado: 19/06/06 Reenviado: $27 / 10 / 06$ Aprovado: 04/11/06 